Experimental study of variations in background radiation and the effect on Nuclear Car Wash sensitivity

J.A. Church, D.R. Slaughter, E.B. Norman, S. Asztalos, P. Biltoft, M.-A.Descalle, J. Hall, T. Luu, D.R. Manatt, G.J. Mauger, D. Petersen, S. Prussin

March 16, 2007 
This document was prepared as an account of work sponsored by an agency of the United States Government. Neither the United States Government nor the University of California nor any of their employees, makes any warranty, express or implied, or assumes any legal liability or responsibility for the accuracy, completeness, or usefulness of any information, apparatus, product, or process disclosed, or represents that its use would not infringe privately owned rights. Reference herein to any specific commercial product, process, or service by trade name, trademark, manufacturer, or otherwise, does not necessarily constitute or imply its endorsement, recommendation, or favoring by the United States Government or the University of California. The views and opinions of authors expressed herein do not necessarily state or reflect those of the United States Government or the University of California, and shall not be used for advertising or product endorsement purposes.

This work was performed under the auspices of the U.S. Department of Energy by University of California, Lawrence Livermore National Laboratory under Contract W-7405-Eng-48. 


\title{
Experimental study of variations in background radiation and the effect on Nuclear Car Wash sensitivity
}

\author{
J.A. Church ${ }^{\mathrm{a}}$, D.R. Slaughter ${ }^{\mathrm{a}}$, E.B. Norman ${ }^{\mathrm{a}, \mathrm{b}}$, S. Asztalos ${ }^{\mathrm{a}}$, \\ P. Biltoft ${ }^{a}$, M.-A. Descalle ${ }^{\text {a }}$, J. Hall ${ }^{\text {a }}$, T. Luu ${ }^{\text {a }}$, D. Manatt ${ }^{\text {, }}$, \\ J. Mauger $^{\mathrm{a}}$, D. Petersen ${ }^{\mathrm{a}, \mathrm{b}}, \mathrm{S}$. Prussin ${ }^{\mathrm{b}}$ \\ ${ }^{a}$ Lawrence Livermore National Laboratory, 7000 East Avenue, Livermore, CA \\ 94550 \\ ${ }^{\mathrm{b}}$ University of California, Berkeley, Berkeley, CA 94720
}

\begin{abstract}
Error rates in a cargo screening system such as the Nuclear Car Wash [1-7] depend on the standard deviation of the background radiation count rate. Because the Nuclear Car Wash is an active interrogation technique, the radiation signal for fissile material must be detected above a background count rate consisting of cosmic, ambient, and neutron-activated radiations. It was suggested previously $[1,6]$ that the variation in this background rate exceeds a purely statistical (Poisson) fluctuation. Corresponding negative repercussions for the sensitivity of the system were shown. Therefore, to assure the most accurate estimation of the variation, experiments have been performed to quantify components of the actual variance in the background count rate, including variations in generator power, irradiation time, and container contents. The background variance is determined by these experiments to be a factor of 2 smaller than values assumed in previous analyses, resulting in substantially improved projections of system performance for the Nuclear Car Wash.
\end{abstract}

\section{Introduction}

In the fight against terrorism at our nation's ports, active cargo container interrogation can detect the presence of fissile material in the assessment of a possible threat. At Lawrence Livermore National Laboratory, one such system 'the Nuclear Car Wash' [1-5] has undergone feasibility testing. The system employs active neutron interrogation, using neutrons to induce fission, and subsequent $\beta$-delayed high-energy $\gamma$-rays as the signal that fissile material has been stowed in the cargo. In some cases, the decay of delayed neutrons is 
used in conjunction with that of the delayed $\gamma$-rays as this signature. Because the Nuclear Car Wash is an active interrogation technique, the background radiation for the system consists not only of cosmic and ambient radiation, but also includes radiation from nuclear species in the cargo or surrounding structures that have been activated by the incident neutron flux.

A system prototype has been set up at LLNL to facilitate tests with hydrogenous and metallic cargoes (see Ref. [6]). Previous experiments designed to test the effects of incident neutron attenuation show that the method is successful, and has the potential to play a major role in reducing the false alarm rates in any combined-method scanning apparatus that may be implemented at the seaports $[1,6]$. In those experiments, the standard deviation of the background count rate distribution was determined to be twice the Poisson deviation folded with another $15 \%$ to accomodate scaling of the data by the generator power. While the error rates reported did indeed seem low, they were artificially higher than actual values due to this conservative estimation for the standard deviation, and gave a false sense of the sensitivity of the system.

$95 \%$ success rate and $0.1 \%$ false alarm rates have been identified as goals for the system [2], both of which depend on the standard deviation of the background count rate distribution. In order to improve the projected error rates, further experiments have been performed to quantify the background count rate distribution in the presence of changing environmental factors. Understanding the contributions to the background count rate caused by varying neutron generator power, irradiation time, and varying container contents allows a more accurate determination of the overall standard deviation of the background count rate, and results in a reduction in the standard deviation from the value that was previously reported $[1,6]$. In this way, the overall sensitivity of the Nuclear Car Wash is more accurately determined and shown to be better than previously reported.

\section{$2 \beta$-delayed $\gamma$-rays with $\mathbf{E}_{\gamma} \geq 3 \mathrm{MeV}$ signal fissile material}

The Nuclear Car Wash uses $\beta$-delayed radiation to signal the presence of fissile material in cargo. It has been shown that after thermal-neutron induced fission, $\beta$-delayed $\gamma$-rays with $\mathrm{E}_{\gamma} \geq 3 \mathrm{MeV}$ are a unique signature for ${ }^{235} \mathrm{U}$ and ${ }^{239} \mathrm{Pu}$ [7]. Many of these fission products have half-lives ranging $1 \mathrm{~s} \leq \mathrm{t}_{1 / 2}$ $\leq 30 \mathrm{~s}$, indicating a chance for many decays within one minute. They also have high $\mathrm{Q}$-values to $\beta$-decay [8], meaning an equally important chance for emission of a high-energy $\gamma$-ray, which because of its energy, will avoid full attenuation by the cargo. While the number of high energy $\gamma$-rays emitted by a particular fission product per fission may be small, the sum of these 


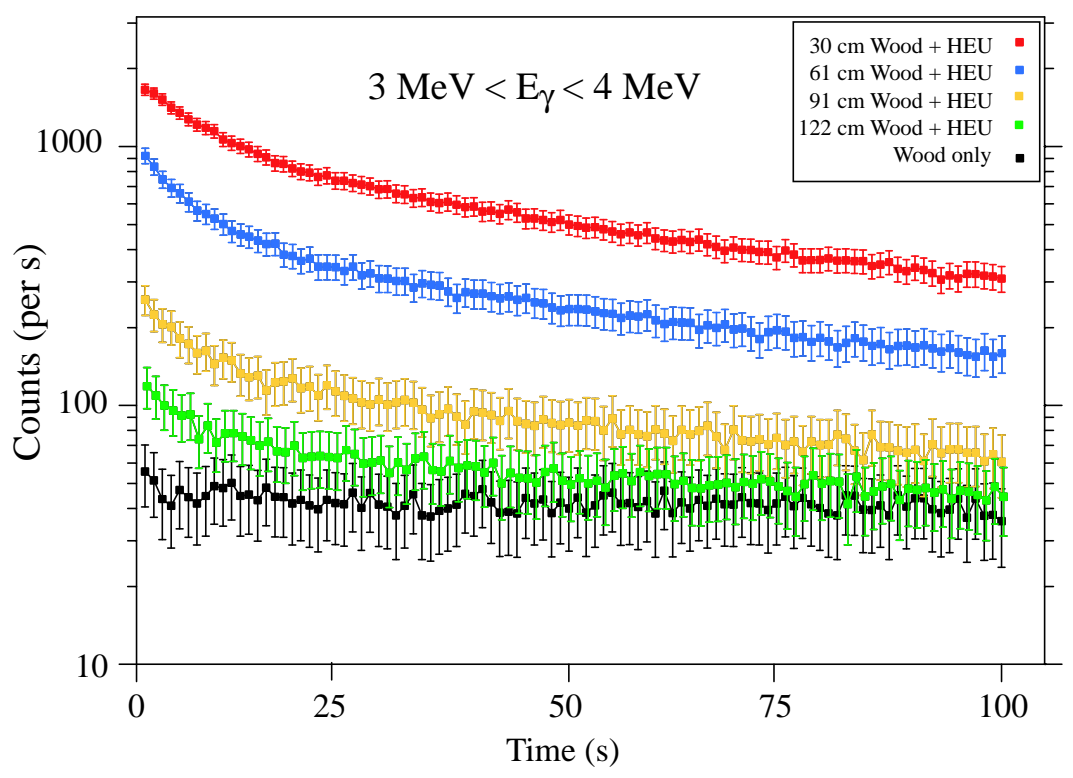

Fig. 1. Decay curves for HEU in wood after a single $30 \mathrm{~s} \mathrm{n}$ irradiation. Distances correspond to the thickness of wood between the floor and the HEU sample. Data has been scaled to a $25 \mu \mathrm{A}$ deuterium current.

$\gamma$-rays over the wide mass distribution of fission products is large enough to produce a detectable signal even in the presence of dense cargo. (For reference, 0.127 delayed $\gamma$-rays with energy above $3 \mathrm{MeV}$ and 0.015 delayed neutrons are emitted per fission for thermal fission in ${ }^{235} \mathrm{U}$ [8].) Their attenuation at $\mathrm{E}_{\gamma} \geq$ $3 \mathrm{MeV}$ is small even in thick cargoes, smaller than attenuation at any other energy. Consequently, and because significant natural background $\gamma$-radiation falls below $2.6 \mathrm{MeV}$, this summed number of high-energy $\beta$-delayed $\gamma$-rays escaping the cargo becomes a unique and abundant signal for the presence of fissile material in thick cargo.

In practice, the alarming signal is obtained by measuring $\gamma$-ray energy and intensity while preserving time information. $\gamma$-ray energy and intensity recorded in time bins are utilized to produce a decay curve for an energy window of choice. In the presence of $\beta$-decaying species with half-lives less than or comparable to the counting time duration, the curve will show an exponential decay in the number of $\gamma$-rays with energies inside the chosen window (Fig 1 ). For counting time durations of $100 \mathrm{~s}$ or less, and $\mathrm{E}_{\gamma} \geq 2.6 \mathrm{MeV}$, it is expected that the majority of the $\gamma$-rays result from $\beta$-decaying species which have been activated by the incident neutrons. Moreover, activated background species other than ${ }^{19} \mathrm{~F}$ are expected to have specific activities a factor of $10^{5}$ less than that of ${ }^{19} \mathrm{~F}(\mathrm{n}, \alpha){ }^{16} \mathrm{~N}$ (see Ref. [6]) or, the resulting product half-lives are much longer than $100 \mathrm{~s} .{ }^{56} \mathrm{Mn}$ for example, is an activation product from steel, with a half-life of $2.5 \mathrm{~h}$. The result, without the presence of decaying fission products or ${ }^{16} \mathrm{~N}$, is that the decay curve is essentially flat (See 'wood only' in Fig 1). Potential change in background count rate caused by the buildup 

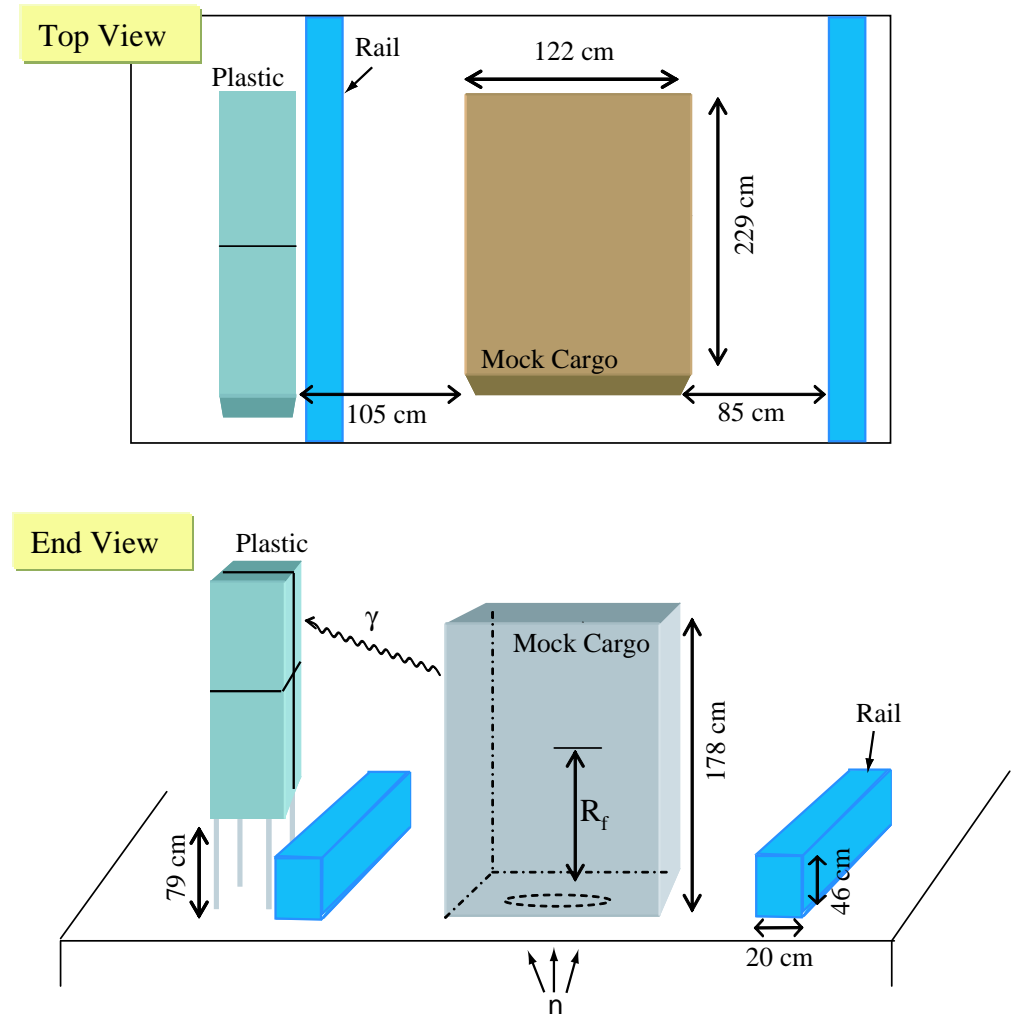

Fig. 2. Experiment dimensions.

of longer-lived species over a day of scanning will be analyzed below.

3-dimensional plots are also constructed from the energy, timing and intensity information by the alarming algorithm ALARMA [9], which is under development but beyond the scope of this report. The $\gamma$-ray energy window chosen in past reports has been approximately $3 \mathrm{MeV} \leq \mathrm{E}_{\gamma} \leq 5 \mathrm{MeV}$ due to considerations mentioned above. Changes in signal to background ratios would occur should the window be altered, especially by decreasing the lower bound. Effects of changing this window on the variation in the background count rate is beyond the scope of this report but should also be examined.

\section{Experiment setup}

The experiment setup (Fig. 2) consists of a broad spectrum 3-7 MeV neutron source, four large area plastic scintillators for $\gamma$-ray detection, and a trolley system to simulate an in-port scanning system. Both $0.55 \mathrm{~g} / \mathrm{cm}^{3}$ mock hydrogenous cargo (plywood), and a $0.6 \mathrm{~g} / \mathrm{cm}^{3}$ mock metallic cargo (steel pipes) were used in conjunction with a standard $610 \times 244 \times 244 \mathrm{~cm}(20 \times 8 \times 8 \mathrm{ft}$.) steel cargo container (not shown). These densities are greater than $98 \%$ of the 
cargo shipped into the U.S. as determined by the PIERS database [11]. The dimensions of the mock cargoes are $229 \mathrm{~cm} \times 122 \mathrm{~cm} \mathrm{x} 178 \mathrm{~cm}$.

Neutrons are produced in a $\mathrm{d}(\mathrm{d}, \mathrm{n})$ reaction. A $4 \mathrm{MeV}$ deuteron linac from Accsys Technologies (Pleasanton, CA) accelerates deuterons horizontally to 4 $\mathrm{MeV}$. The deuteron beam direction is then bent 90 degrees upward by a high energy beam transport system and is delivered to the 1 atm, $60 \mathrm{~cm}$ long, $\mathrm{D}_{2}$ gas target cell at currents of $10-65 \mu \mathrm{A}$ nominally (100 $\mu \mathrm{A}$ maximum). A $10 \mu \mathrm{m}$ Mo window slows the beam to approximately $3.27 \mathrm{MeV}$ upon entry into the cell where the incident deuterons are completely stopped. Neutrons resulting from the $d(d, n)$ reaction are forward focused [5] and allowed to escape through the floor of the high bay laboratory via a polyethylene collimator with a 15 degree opening half angle.

The background experiments are performed by first cycling the neutron generator on to activate background activation species. The plastic detectors are shuttered off during this time. Neutrons are then turned off, and the plastic detectors are allowed to count, recording time and energy information via standard NIM electronics for construction of decay curves after the data acquisition. Each plastic detector has dimensions of $61 \times 61 \times 25.4 \mathrm{~cm}$, and an energy resolution of approximately $35 \%$ at $898 \mathrm{keV}$, and $13 \%$ at $6000 \mathrm{keV}$. Unless otherwise indicated, data will be shown as decay curves created from software-sums of the individual spectra over a defined range of energy depositions in the plastic detectors.

For the purpose of this study, the experiment configuration, irradiation and counting times were varied slightly for each parameter measurement. The configurations are described measurement-by-measurement in the sections below.

\subsection{Plastic scintillator calibration}

To achieve the energy calibration for the plastic detectors, they are first gainmatched by adjusting the high voltage and shaper settings to reach a linear response region in each of the detectors (see Ref. [10]) and match the dispersion of the pulse height spectra for each of the four detectors. For this purpose, and at the start and end of each day, a $10 \mu \mathrm{Ci}{ }^{88} \mathrm{Y}$ source ( 898 and $1836 \mathrm{keV}$ ) is placed at the horizontal and vertical center of the four detectors, $115 \mathrm{~cm}$ away from the detectors toward the neutron aperture (see Fig. 2). In order to include an accurate respresentation at the energies above $3 \mathrm{MeV}$ pertinent to this experiment, a sample of Teflon is also placed above the neutron aperture and irradiated in order to induce the ${ }^{19} \mathrm{~F}(\mathrm{n}, \alpha){ }^{16} \mathrm{~N}$ reaction, facilitating $\beta$-decay of ${ }^{16} \mathrm{~N}$ to ${ }^{16} \mathrm{O}$, and the subsequent emission of a $6.1 \mathrm{MeV} \gamma$-ray. Compensation is made for the incomplete energy collection in the plastic by assuming maximum 


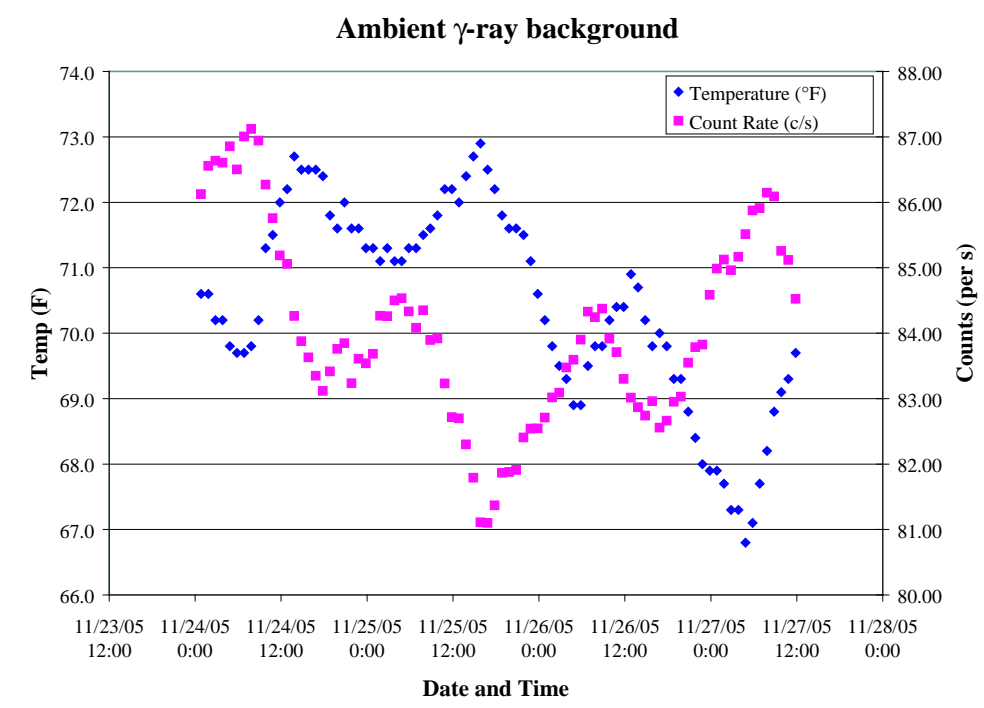

Fig. 3. Ambient $\gamma$-ray background measured with one $25.4 \mathrm{~cm}$ thick plastic scintillator. $\gamma$-rays with energies between 3 and $6 \mathrm{MeV}$ were histogrammed every hour over the course of 4 days. Average number of counts per second for the hour time bins are plotted in conjunction with air temperature [10].

energy depositions corresponding to:

$$
E_{\text {peak }}=E_{\gamma}-\left(\left(E_{\gamma}-E_{\text {compton }}\right) / 2\right),
$$

as determined via monte carlo simulation [12]. A linear fit of the channel vs. energy curve is used to produce energy calibration coefficients. The same procedure was followed in Ref. $[1,6]$.

\section{$4 \quad$ Fluctuations in ambient $\gamma$-ray background}

The detected ambient background count rate for the system was studied in order to understand its long-term fluctuation with temperature as well as fluctuations over a typical 3 cycle experimental run (three $100 \mathrm{~s}$ count times separated by $30 \mathrm{~s}$ wait times to mimic irradiation measurements). Experiments were performed with nothing over the neutron aperture, and the cargo container moved away from the detector area. The background count rate was found to be steady over a short time, but varied with temperature fluctuations at longer time scales.

Figure $3[10]$ shows ambient $\gamma$-rays depositing energies from 3 to $6 \mathrm{MeV}$, measured in one hour segments with one $25.4 \mathrm{~cm}$ thick plastic detector over the 
Ambient Background Detector 4D- one cycle

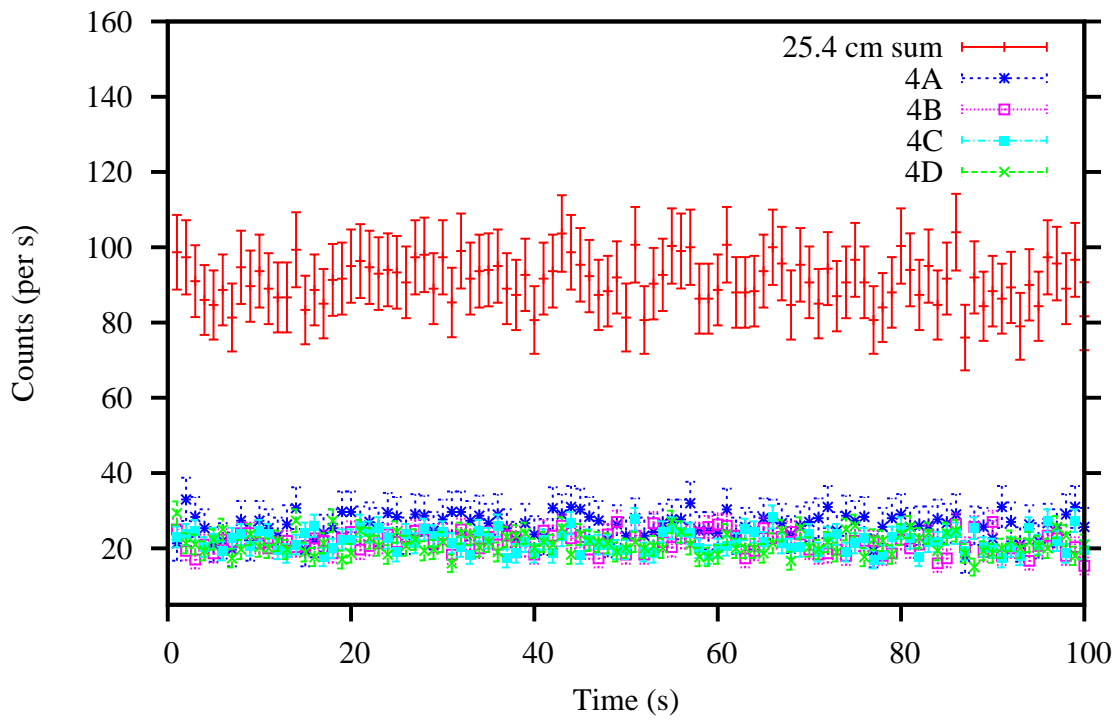

Fig. 4. Decay curves representing a background measurement made prior to irradiations are shown for a $\gamma$-ray energy window from 3 to $5 \mathrm{MeV}$. One $100 \mathrm{~s}$ counting cycle is represented for each of four 25.4 $\mathrm{cm}$ thick detectors- $4 \mathrm{~A}, 4 \mathrm{~B}, 4 \mathrm{C}$ and $4 \mathrm{D}$. The sum of the four signals is also shown.

course of 4 days. No neutron irradiation experiments were carried out during these four days. A general anti-correlation with temperature can be seen for the average number of counts per second for each hour long measurement. After PMT stabilization (at approximately 1200 hrs on 11/24/06), an $8.2 \%$ decrease in room temperature results in a $6.2 \%$ increase in the number of ambient background $\gamma$-rays detected per second, averaged over the one hour counting time.

Figure 4 shows ambient $\gamma$-ray backgrounds measured in all four of the 25.4 cm detectors ('4A,B,C,D'). The measurement was made prior to any neutron irradiations. $\gamma$-rays depositing energies between 3 and $5 \mathrm{MeV}$ were counted in $1 \mathrm{~s}$ time bins for a total of $100 \mathrm{~s}$. There is no variation in the detected ambient background count rate over $100 \mathrm{~s}$.

Background measurements taken after the completion of a set of irradiation measurements are referred to as 'passive' background measurements. No irradiations are made during the actual passive background measurement, but irradiations have occurred at some point prior to its start. A completely 'ambient' background (no prior irradiations) differs from a 'passive' background (prior irradiations), in that the passive background may have contributions from longer-lived species that were activated during the previous irradiations. Panel B of Fig. 6 demonstrates the stability of the passive background count rate over a 3 cycle counting period, in which 100 s counting cycles are per- 
Passive Background 4- 10" detectors- one cycle, 3-5 MeV

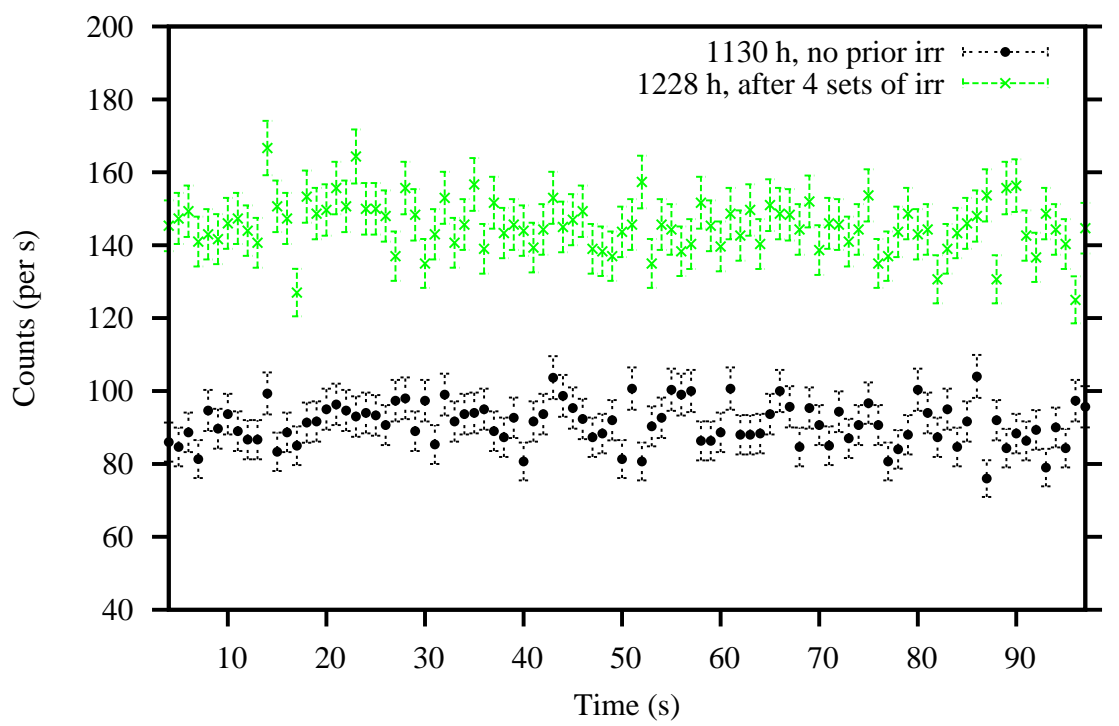

Fig. 5. Passive $\gamma$-ray background measurements made before and after neutron irradiations totalling $360 \mathrm{~s}$ over one hour. $\gamma$-rays depositing between 3 and $5 \mathrm{MeV}$ in four $25.4 \mathrm{~cm}$ thick detectors are shown in 1 $\mathrm{s}$ time bins for a counting cycle of $100 \mathrm{~s}$.

formed after $30 \mathrm{~s}$ wait times. Over the course of $390 \mathrm{~s}$, the passive background level again does not change.

Because passive backgrounds are taken directly before and after the irradiation for these exploratory experiments, the implication here is that the variation in the passive background count rate has no effect beyond statistical considerations on the standard deviation of the overall $\gamma$-ray background count rate. Furthermore, the same statement can be applied to the alarm algorithm ALARMA [9]. The algorithm uses the last 10 seconds (for example) of the count time in question to determine the background count rate. Because the passive background is steady over the entirety of a typical 100 s count time, the method employed by ALARMA is assuming an accurate passive background rate for the count cycle. Over a long series of irradiations, ALARMA considers the most pertinent passive background for the measurement.

\section{The buildup of $\gamma$-ray background due to multiple neutron irra- diations}

Due to the activation of longer-lived species in the surrounding environment, the overall level of $\gamma$-ray background count rate will increase with increasing number of neutron irradiations of the same cargo or experiment configuration. 


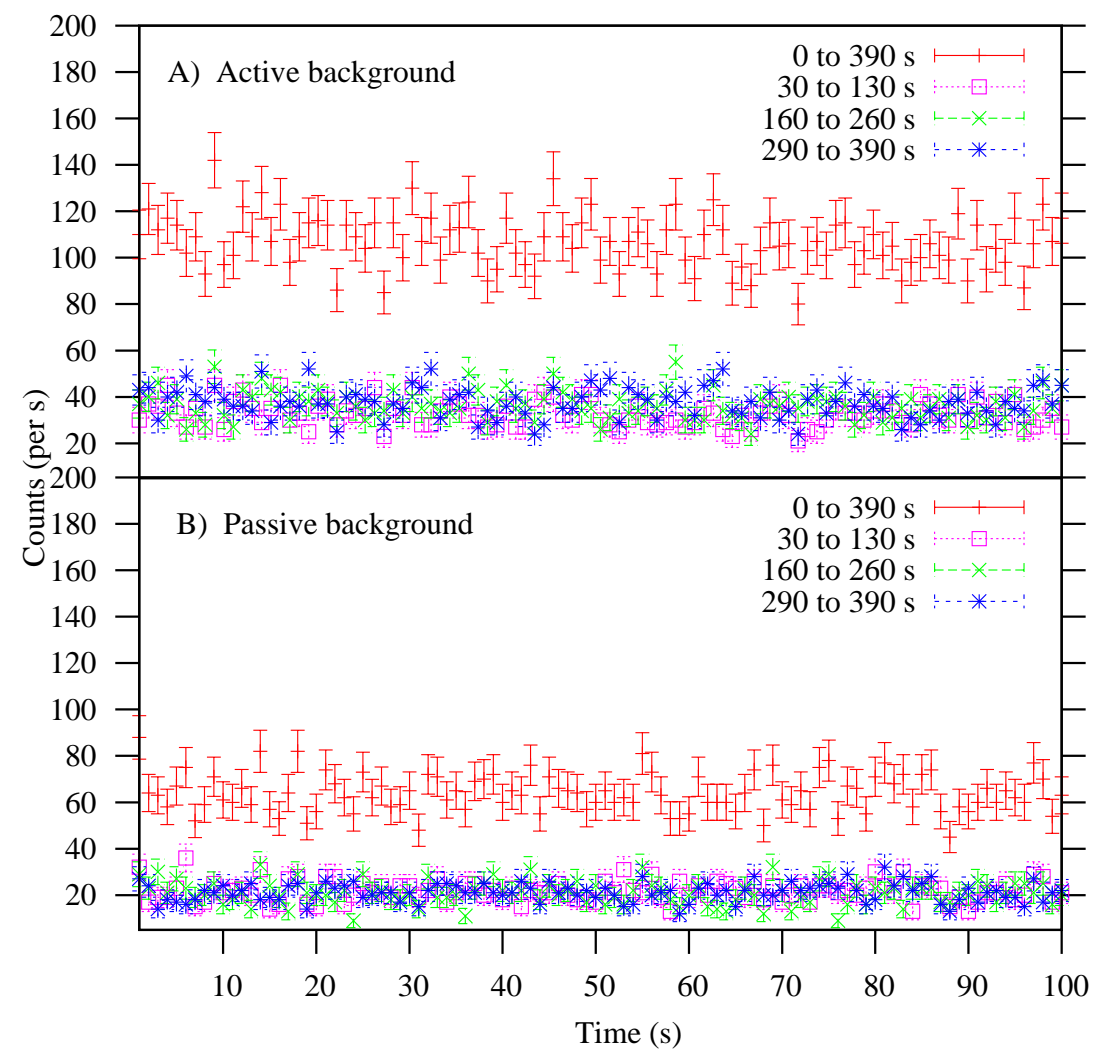

Fig. 6. Panel A) $\gamma$-rays depositing energies between 3 and $5 \mathrm{MeV}$ in one $25.4 \mathrm{~cm}$ thick plastic scintillator are counted in $1 \mathrm{~s}$ time intervals for $100 \mathrm{~s}$ after each of three $30 \mathrm{~s}$ neutron irradiations with a deuterium current of $32 \mu \mathrm{A}$. Between neutron irradiations, the background rate does not change. Panel B) is a parallel experiment performed without neutron irradiation demonstrating the constant ambient background over the same time scale.

For example, two of the constituents of steel, ${ }^{56} \mathrm{Fe}$ and ${ }^{55} \mathrm{Mn}$, can undergo the reactions ${ }^{56} \mathrm{Fe}(\mathrm{n}, \mathrm{p}){ }^{56} \mathrm{Mn}$ and ${ }^{55} \mathrm{Mn}(\mathrm{n}, \gamma){ }^{56} \mathrm{Mn}$ respectively at the neutron energies utlized in our experiments. ${ }^{56} \mathrm{Mn}$ decays with a half-life of 2.58 hours emitting a $2.113 \mathrm{MeV} \gamma$-ray (14\%) and 5 others with energies from $2.5 \mathrm{MeV}$ to $3.4 \mathrm{MeV}$ at smaller fractions. Because of the poor energy resolution of the plastic detectors $(\geq 13 \%$ at $6 \mathrm{MeV})$, these $\gamma$-rays could be included in an energy window defined by a lower limit of $3 \mathrm{MeV}( \pm 7 \%)$. Another candidate is the ${ }^{48} \mathrm{Ca}$ constituent of wood which can undergo ${ }^{48} \mathrm{Ca}(\mathrm{n}, \gamma){ }^{49} \mathrm{Ca} .{ }^{49} \mathrm{Ca}$ decays with a half-life of $8.72 \mathrm{~min}$., emitting a $3.084 \mathrm{MeV} \gamma$-ray, which would easily be included in an energy window above $3 \mathrm{MeV}$. Both wood and steel are present in the experiment setup. There are steel floor plates over the neutron generator, steel rails for the trolley system, and wood is part of the floor of the cargo container itself. 
Figure 5 shows the change between ambient and passive background count rates over the course of four experimental sets of three irradiation cycles each, $360 \mathrm{~s}$ of neutron irradiation in total, spaced over one hour. The generator power was different for each of the 4 sets, $53 \mathrm{~W}$ (3 cycles), $90 \mathrm{~W}$ (6 cycles (2 sets) ) and $126 \mathrm{~W}$ (3 cycles). The cargo container was parked away from the detector wall, and nothing was placed over the neutron aperture for these measurements. The result was approximately a $50 \%$ increase in the overall background count rate after 4 sets of irradiation cycles due to the activation of longer lived species as discussed above.

On a $100 \mathrm{~s}$ time scale, previous results [6] indicate that, except for ${ }^{19} \mathrm{~F}$, there seems to be no other short-lived (decaying with a half-life between 1 and $30 \mathrm{~s}$ ) isotope in the chart of the nuclides likely to produce a significant interference to a $\gamma$-ray signal with energies above $3 \mathrm{MeV}$. Figure 6 shows that the background rate is indeed steady to within statistical variation between neutron bursts for an experiment of three $30 \mathrm{~s}$ irradiations followed by $100 \mathrm{~s}$ counting times. Because the difference in the background rate before the first burst and after the last burst is negligible, the contribution to the standard deviation of the overall background count rate due to multiple irradiations can be taken to be no greater than the statistical variation if a passive background rate taken directly before or after the measurement is used.

\section{Fluctuations in the n-induced $\gamma$-ray background due to varying generator power}

It has been assumed in previous [1,6] analyses, that the variation in the active background count rate resulting from varying generator powers between complete measurements was greater than one Poisson standard deviation. That conclusion was drawn with a rough estimation utilizing active background measurements made throughout the course of the experimental campaign which were not designed solely for the determination of the variation due to a change in generator power. Here we have performed a set of measurements designed solely for this purpose.

Figure 7 shows a set of background measurements made consecutively with deuterium currents of $23 \mu \mathrm{A}$ at $1156 \mathrm{~h}, 32 \mu \mathrm{A}$ at $1213 \mathrm{~h}$, and $13 \mu \mathrm{A}$ at 1220 h. One set of three $30 \mathrm{~s} 23 \mu \mathrm{A}$ irradiations of a Teflon sample was made between the $23 \mu \mathrm{A}$ active background measurement and the $32 \mu \mathrm{A}$ active background measurement. Panel A of Fig. 7 shows all three active background measurements along with the ambient background count rate taken prior to any irradiation and one passive background count rate taken after all of the irradiations. 


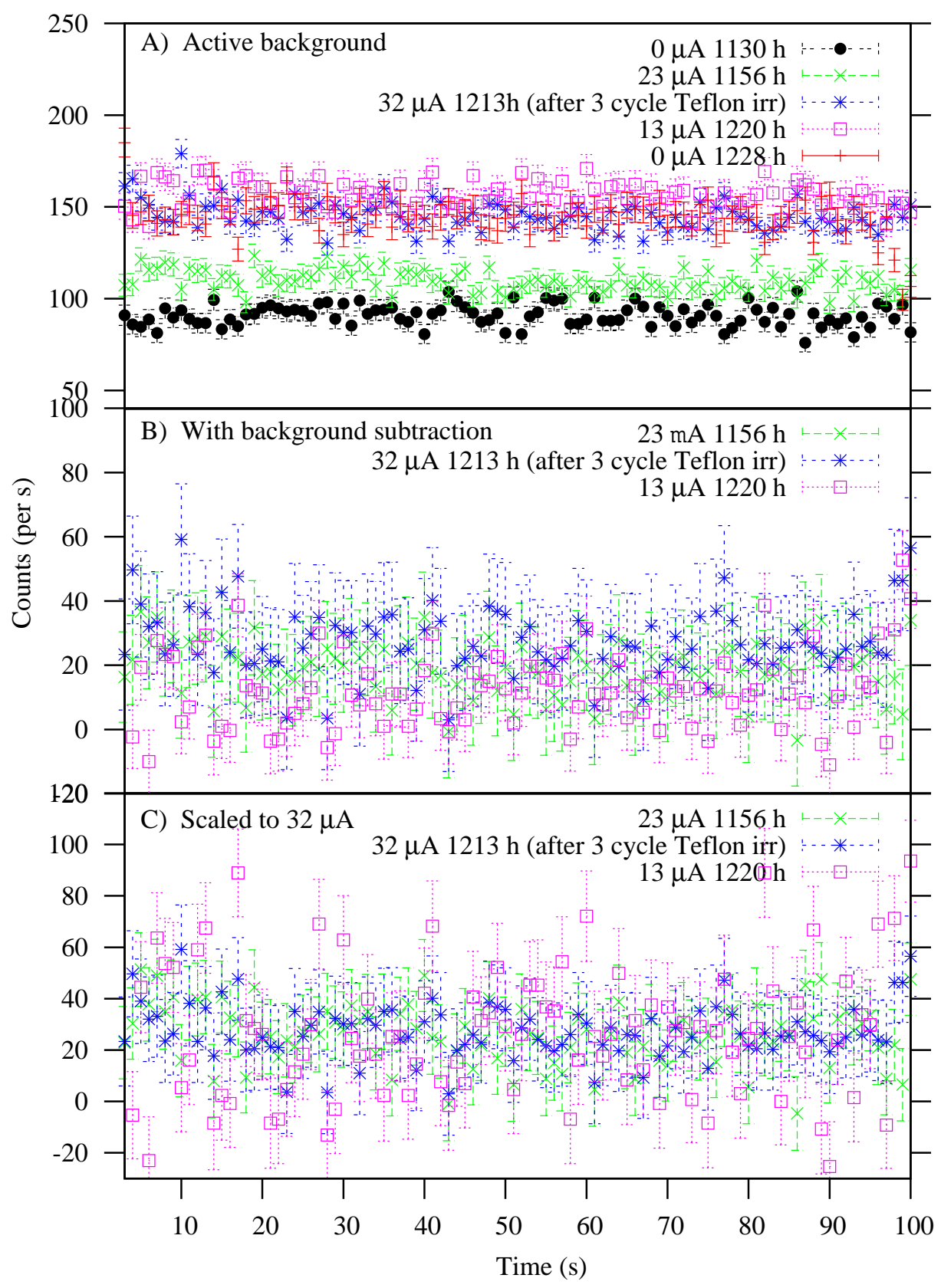

Fig. 7. Active $\gamma$-ray background count rate for varying deuterium power into the $\mathrm{D}_{2}$ gas cell. The sum of four $25.4 \mathrm{~cm}$ thick detectors is represented. The decay curves include $\gamma$-rays depositing energies from 3 to $5 \mathrm{MeV}$ in the plastic.

To compare the fluctuation caused solely by the varied generator power, the appropriate passive background rate must be subtracted from the active background measurements. Passive background count rates were measured directly before the $23 \mu \mathrm{A}$ active background measurement and directly after the $13 \mu \mathrm{A}$ 


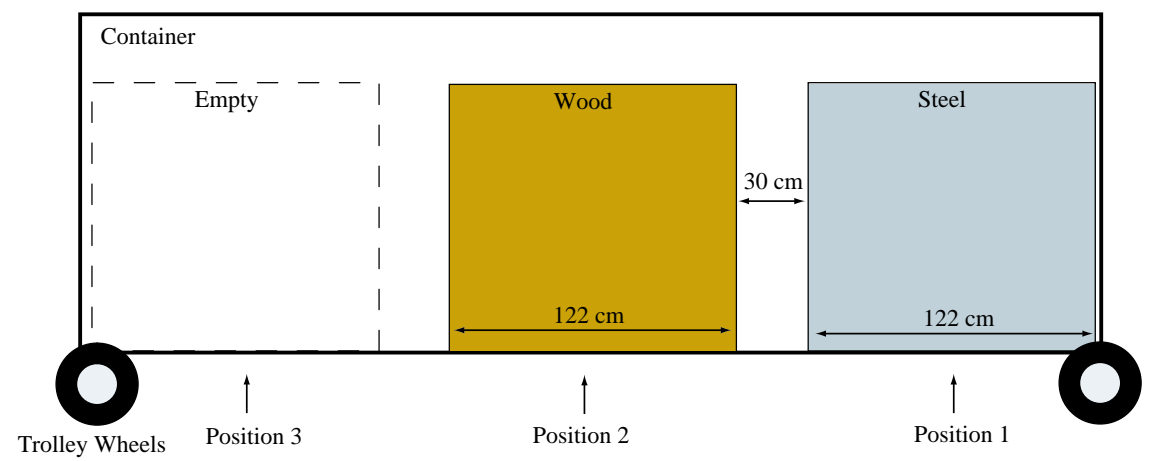

Fig. 8. The cargo configuration within a standard $610 \mathrm{~cm}(20 \mathrm{ft})$ long steel cargo container. Position 1,2, and 3 indicate the location of the center of the neutron aperture for different experiments as explained in the text. The wheels are present, but not pertinent to the experiments described here.

measurement, and each of these (shown in Panel A of Fig. 7) were subtracted accordingly. In order to estimate the passive background for the $32 \mu \mathrm{A}$ measurement, the average of the two other passive backgrounds was taken. The results of the passive background subtraction are shown in Panel B of Fig. 7.

As a final step for the comparison, each measurement was scaled to a generator power of $32 \mu \mathrm{A}$ (Panel C of Fig. 7). The wide dispersal of the $13 \mu \mathrm{A}$ scaled data is due to the scaling of negative numbers after the background subtraction. At this low power the active background rate is not significantly greater than that of the passive background. However, the averages of the backgroundsubtracted scaled data can still be compared. Background-subtracted averages without scaling are: $19(14) \mathrm{c} / \mathrm{s}$ for the $23 \mu \mathrm{A}$ measurement, 27(16) for the 32 $\mu \mathrm{A}$ measurement and 11(17) for the $13 \mu \mathrm{A}$ measurement. After scaling to 32 $\mu \mathrm{A}$ the averages are: $26(14) \mathrm{c} / \mathrm{s}$ for the $23 \mu \mathrm{A}$ measurement, 27(16) for the 32 $\mu \mathrm{A}$ measurement and 26(17) for the $13 \mu \mathrm{A}$ measurement. After scaling to the highest generator power, the active background rates are all within 1 Poisson standard deviation of one another. This is a factor of 2 improvement over the previous estimation for the variation in the background fluctuation caused by changes in the generator power.

\section{Background experiments within a cargo container}

Experiments were also performed to study the dependence of the $\gamma$-ray background rate on irradiation time and variation of cargo load. The experiments were accomplished by placing the $0.55 \mathrm{~g} / \mathrm{cm}^{3}$ plywood mock cargo and the 0.6

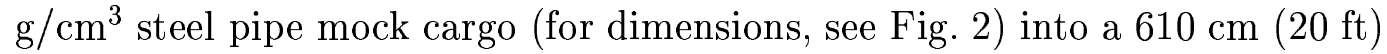
long standard steel cargo container $(610 \times 244 \times 244 \mathrm{~cm})(20 \times 8 \times 8 \mathrm{ft})$. The 
placement of the mock cargoes inside the container is illustrated in Fig. 8.

Measurements of the loaded cargo container were made at each position (neutron aperture centered below steel (Position 1), wood (Position 2) and air (Position 3)) for irradiations times of 3, 10 and 30 s. For each position, three cycles neutron irradiation and $100 \mathrm{~s}$ counting were made for each different irradiation time. The deuteron current into the gas cell was $13 \mu \mathrm{A}$ for each of the measurements. Between sets of irradiations, 2 cycles of passive background measurements were taken in the same time configuration $(3,10$ and $30 \mathrm{~s}$ of mimicked irradiation time followed by $100 \mathrm{~s}$ counting periods). When each set of irradiation cycles and passive counting cycles was completed, the container was moved to the next position, and the measurements were repeated. Each measurement was made consecutively beginning with $3 \mathrm{~s}$ irradiations at Position 1 (steel) and ending with $30 \mathrm{~s}$ irradiations at Position 3 (air), for a total duration of $1 \mathrm{hr} 34 \mathrm{~min}$.

\subsection{Fluctuation due to length of irradiation time}

Irradiations of 3, 10 and $30 \mathrm{~s}$ were made at each of the cargo container positions: steel, wood and air centered over the neutron aperture. Three cycles of irradiation each followed by $100 \mathrm{~s}$ count periods were performed for each of the irradiation times in a particular position before moving to the next position. In Fig. 9, decay curves have been constructed for each position including the 3, 10 and 30 s irradiation active $\gamma$-ray background count rate for a $\gamma$-ray window between 3 and $5 \mathrm{MeV}$. The passive background rate has not been subtracted, and is displayed in Fig. 10.

At each position, the overall ('active') background rates fall within 1 standard deviation (Poisson) of each other for each of the irradiation times. For example, at Position 1 (steel), the average active background rate is $66(8) \mathrm{c} / \mathrm{s}$ for the $3 \mathrm{~s}$ irradiation, $67(8) \mathrm{c} / \mathrm{s}$ for the $10 \mathrm{~s}$ irradiation, and $76(9) \mathrm{c} / \mathrm{s}$ for the 30 $\mathrm{s}$ irradiation. All of these averages are within 1 standard deviation of one another, and therefore we can say that the background rate does not vary beyond statistical uncertainties between irradiation times of 3, 10 and $30 \mathrm{~s}$. Although not discussed, the same is true of Position 2 (wood) and Position 3 (air), as can be seen in Fig. 9, panels B and C. Discrepancies between the different positions are discussed in the following section.

\subsection{Fluctuation due to variation of cargo load}

Some insight into the effect the cargo load itself has on the fluctuation of the active background may be gained by evaluating the experiment described 
Active background, 3- $25.4 \mathrm{~cm}$ detectors, 3-5 MeV

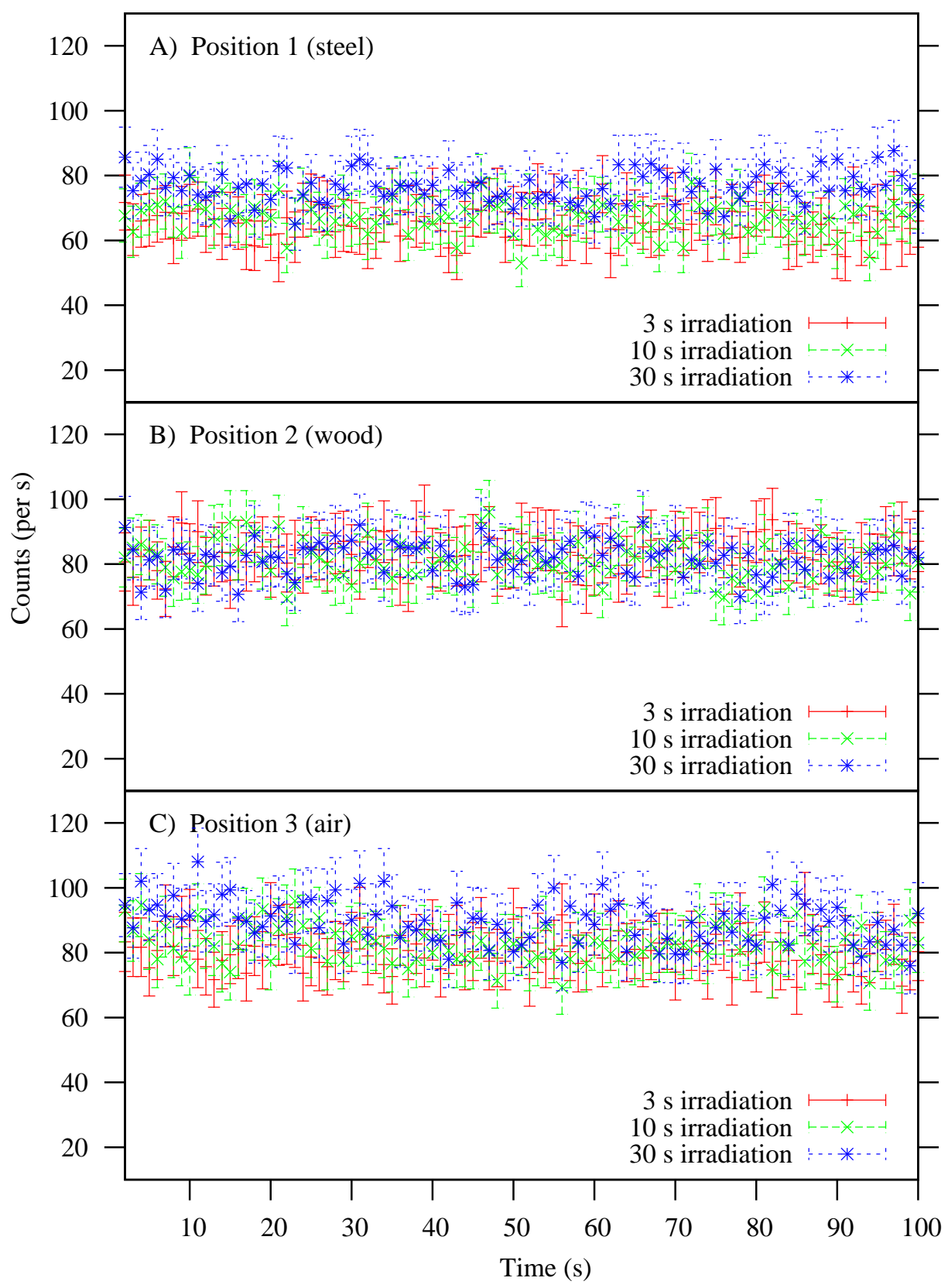

Fig. 9. Active $\gamma$-ray background count rates for $\gamma$-rays depositing energies between 3 and $5 \mathrm{MeV}$ in the sum of three $25.4 \mathrm{~cm}$ thick plastic detectors for neutron aperture center positions (see Fig. 8) A) Position 1, B) Position 2 and C) Position 3. The length of irradiation time is indicated. The deuterium current into the $\mathrm{D}_{2}$ gas cell was 13 $\mu \mathrm{A}$. 
Passive background, 3- $25.4 \mathrm{~cm}$ detectors, 3-5 MeV

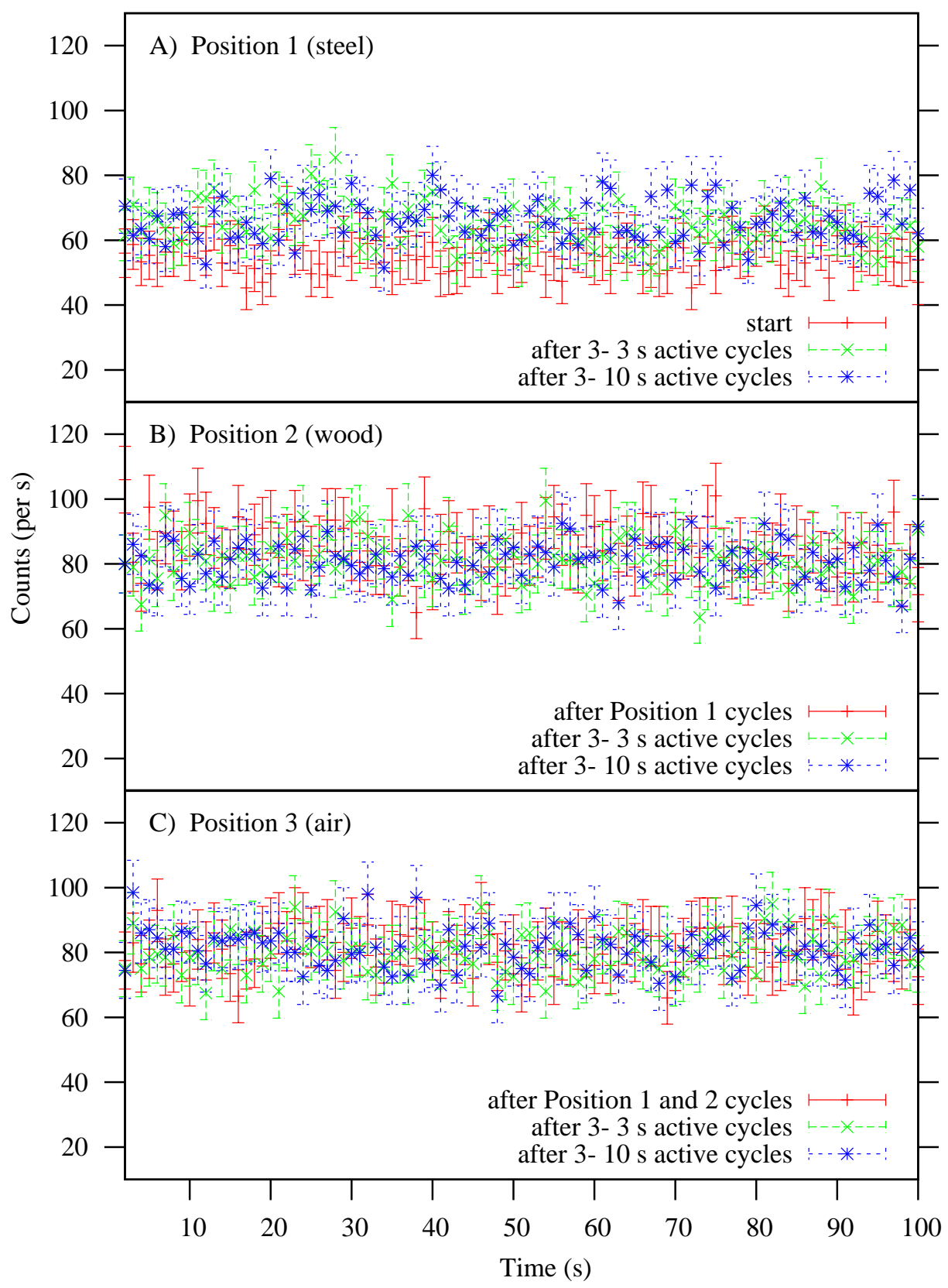

Fig. 10. Passive $\gamma$-ray background count rate for $\gamma$-rays depositing energies between 3 and $5 \mathrm{MeV}$ in the sum of three $25.4 \mathrm{~cm}$ thick plastic detectors for neutron aperture center positions (see Fig. 8) A) Position 1, B) Position 2 and C) Position 3. The irradiations occuring at that position prior to the measurement are indicated. 
in Section 7.1 in a different manner. As described, a different representative cargo was placed at 3 different positions inside a typical cargo container. Each of these were moved in turn to the center of the neutron aperture and were subsequently irradiated for several cycles of differing irradiation time at a deuterium current of $13 \mu \mathrm{A}$. While Figures 9 and 10 presented the resulting decay curves arranged by position in order to directly compare the effects of a change in irradiation time, Figures 11 and 12 display the same data, but arranged by irradiation time, in order to examine the effect the position has on the active background count rate for each of the irradiation times.

Because all of the irradiations for any one position were performed prior to moving the container to the next position, it is necessary to subtract the passive background measured prior to each measurement in order to remove longer-lived effects of the previous irradiations from the examination (see Section 5) and extract the effect of cargo load alone on the variation in the background rate. The background-subtracted decay curves are not shown, and average $\gamma$-ray background count rates are considered for energy depositions in the plastic between 3 and $5 \mathrm{MeV}$. Passive background rates subtracted were measured directly before the measurement in question. Average backgroundsubtracted count rates for the $3 \mathrm{~s}$ irradiations are: 10(12) c/s at Position 1 (steel), $-2(13) \mathrm{c} / \mathrm{s}$ at Position 2 (wood) and $-1(13) \mathrm{c} / \mathrm{s}$ at Position 3 (air). Average background subtracted values for the $10 \mathrm{~s}$ irradiations are: $2(12) \mathrm{c} / \mathrm{s}$ at Position 1, $0(13) \mathrm{c} / \mathrm{s}$ at Position 2, 2(13) at Position 3. Finally, average background subtracted values for the $30 \mathrm{~s}$ irradations are: 10(12) at Position $1,1(13)$ at Position 2, and 8(13) at Position 3. Uncertainties are the result of propagation of statistical uncertainties only.

Comparing the passive-background-subtracted averages gives a sense of the active background rate alone for the measurements at each position. From this it appears that the background rate increases the most at Position 1 , the steel load. The active background for Position 2 is basically unchanged above the passive background, which may be explained by the increase in passive background caused by longer-lived species populated in the steel irradiations, all three sets occurring prior to the wood irradiation, and the subsequent small addition to the active background by the wood itself. Increases also occurred at Position 3, which was devoid of cargo but continued to include irradiation of the steel container and its wood flooring. At all positions, the greatest increase occurred during the $30 \mathrm{~s}$ irradiations, the least during the $3 \mathrm{~s}$ irradiations.

For each of the irradiation times, after appropriate passive background subtraction, the overall background count rate level for $\gamma$-rays depositing energies between 3 and $5 \mathrm{MeV}$ are all within one standard deviation using propagated statistical uncertainties. This indicates that a change in the variation in the $\gamma$-ray background count rate beyond statistical fluctuations is not caused by differing cargo configurations inside the container, so long as the background 
Active background, 3- $25.4 \mathrm{~cm}$ detectors, 3-5 MeV

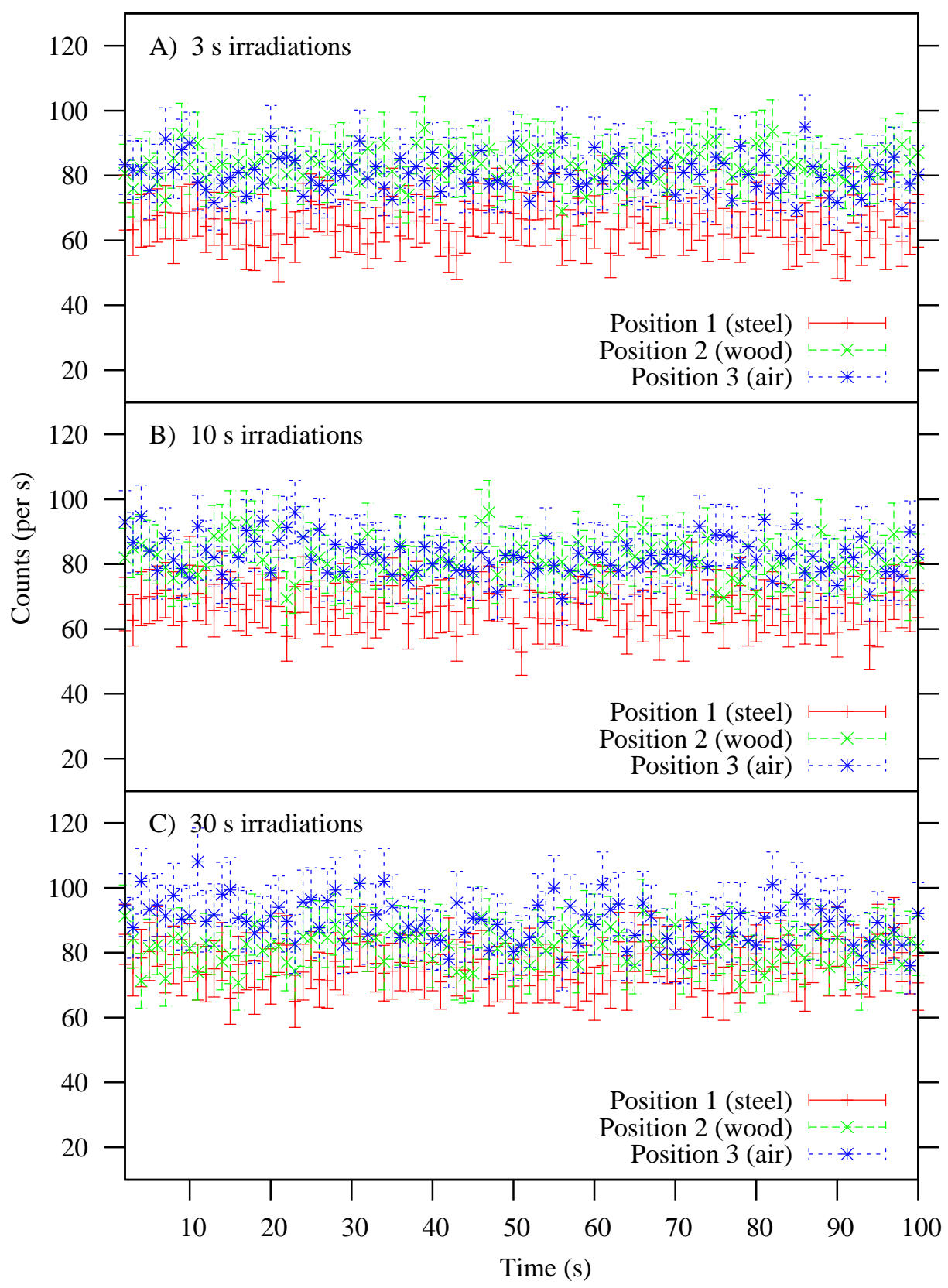

Fig. 11. Active $\gamma$-ray background count rate for $\gamma$-rays depositing energies between 3 and $5 \mathrm{MeV}$ in the sum of three $25.4 \mathrm{~cm}$ thick plastic detectors after A) $3 \mathrm{~s}$ irradiations, B) $10 \mathrm{~s}$ irradiations and C) $30 \mathrm{~s}$ irradiations. The position of the neutron aperture center is indicated (see also Fig. 8). The deuteron current into the $\mathrm{D}_{2}$ gas cell was $13 \mu \mathrm{A}$. 


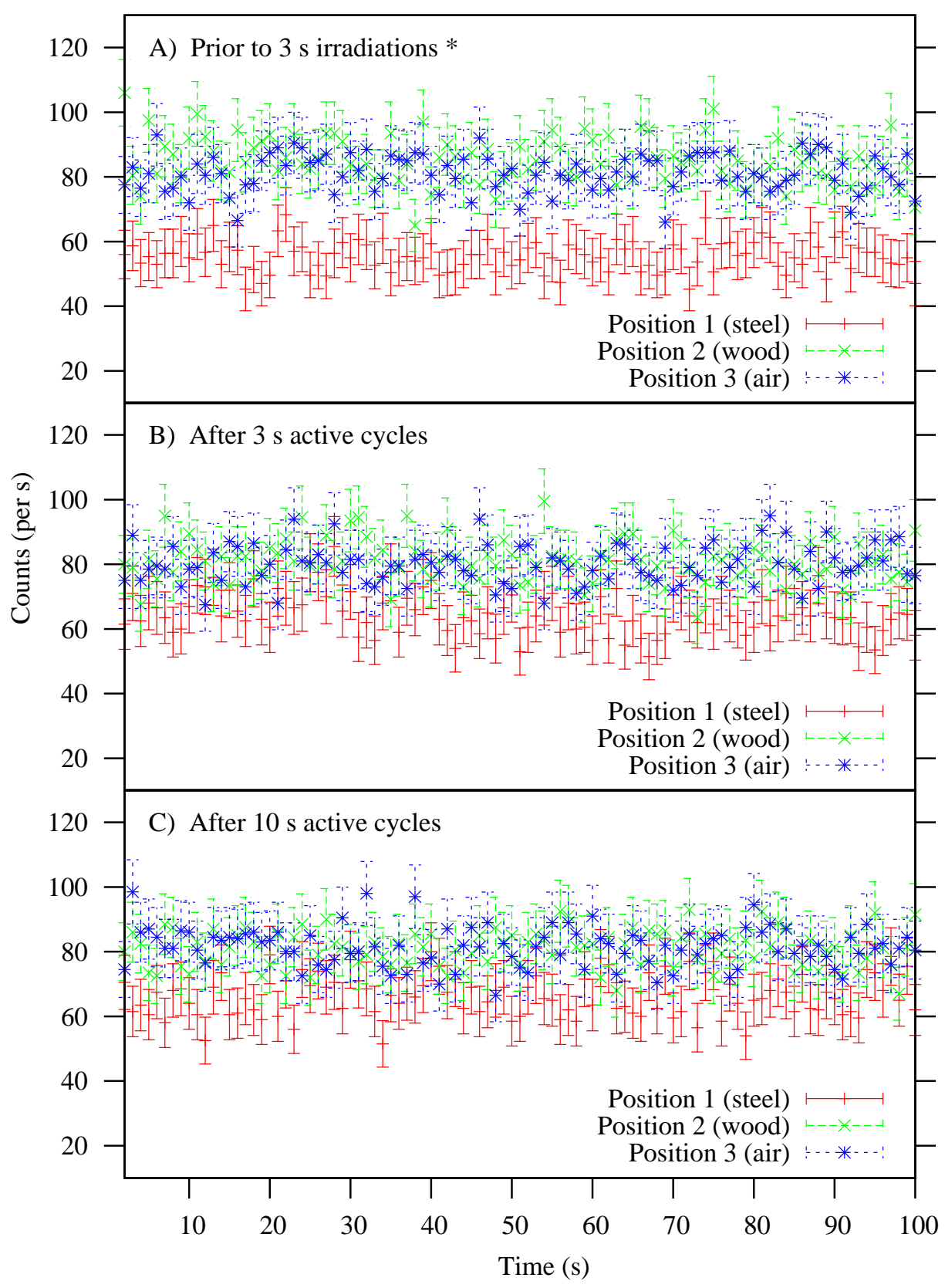

Fig. 12. Passive $\gamma$-ray background rate for $\gamma$-rays depositing energies between 3 and $5 \mathrm{MeV}$ in the sum of three $25.4 \mathrm{~cm}$ thick plastic detectors at the A) start of the irradiations for that position, $\left(^{*}\right.$ the Position 1 measurement is the only one with no prior irradiations to the any part of the system), B) after the $3 \mathrm{~s}$ irradiations and C) after the $10 \mathrm{~s}$ irradiations. The position of the neutron aperture center is indicated (see also Fig. 8) 


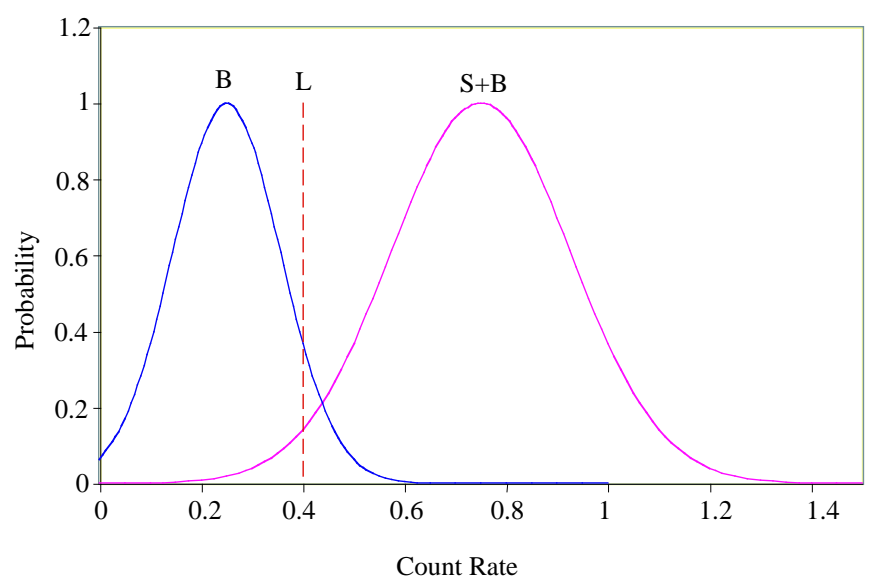

Fig. 13. Sample Gaussian distributions for the background $(B)$ and signal-plus-background $(S+B)$ with an alarm threshold level $L$

count rates are taken directly before or after the measurement in question, as is the method utilized in the alarming algorithm ALARMA [9].

Even without making the background subtraction, the variations at different positions are within one standard deviation (safely within 2) for each irradiation time, again considering values averaged over 100 s. Average count rates (not background-subtracted) including $\gamma$-rays depositing energies between 3 and $5 \mathrm{MeV}$ for $3 \mathrm{~s}$ irradiations are: $66(8) \mathrm{c} / \mathrm{s}$ at Position $1,83(9) \mathrm{c} / \mathrm{s}$ at Position $2,81(9) \mathrm{c} / \mathrm{s}$ at Position 3 . Average count rates for the $10 \mathrm{~s}$ irradiations are: $67(8) \mathrm{c} / \mathrm{s}$ at Position $1,81(9) \mathrm{c} / \mathrm{s}$ at Position 2, and $82(9) \mathrm{c} / \mathrm{s}$ at Position 3. Finally for the $30 \mathrm{~s}$ irradiations average count rates are: $76(9) \mathrm{c} / \mathrm{s}$ at Position $1,81(9) \mathrm{c} / \mathrm{s}$ at Position 2, and $89(9) \mathrm{c} / \mathrm{s}$ at Position 3.

\section{System sensitivity}

Error rates are examined using standard Gaussian probability distributions in the construction of Receiver Operating Characteristic (ROC) curves. Figure 13 is a graphical representation of sample background $(B)$ and signalplus-background $(S+B)$ distributions with an alarm threshold level $L$. The probability for false alarm is given by:

$$
P_{F A}(L, B, \sigma)=\int_{L}^{\infty} \frac{1}{\sqrt{2 \pi} \sigma} e^{-0.5\left(\frac{x-B}{\sigma}\right)^{2}} d x=0.5\left[1-\operatorname{erf}\left(\frac{(L-B)}{\sqrt{2} \sigma}\right)\right] .
$$

Similarly, the expression for detection probability is: 


$$
\begin{aligned}
P_{D}(L, S+B, \sigma) & =\int_{L}^{\infty} \frac{1}{\sqrt{2 \pi} \sigma} e^{-0.5\left(\frac{x-(S+B)}{\sigma}\right)^{2}} d x \\
& =0.5\left[1+\operatorname{erf}\left(\frac{(S+B-L)}{\sqrt{2} \sigma}\right)\right]
\end{aligned}
$$

where $L$ represents the alarm threshold level above which detection is taken to be positive, $B$ is the number of counts in the active background, $S+B$ is the number of signal plus active background counts, and $\sigma$ is the standard deviation in the number of counts in the active background. The ROC curve is constructed by plotting the probability for detection, $P$ (Detection) against the probability for false alarm, $P($ FalseAlarm $)$, where the alarm threshold, $L$, is the independent variable.

In an earlier experimental campaign, feasibility tests were performed with HEU at various depths in plywood and steel pipes. The experimental details can be found in Refs. $[1,6]$. The HEU sample within the cargo was centered over the neutron aperture and the distance $\left(R_{f}\right)$ varied with respect to the floor to test the effects of incident neutron attenuation through the wood. The horizontal distance from the sample to the plastic scintillators was $61 \mathrm{~cm}$, a shorter distance than that of the background measurements presented in this work.

Decay curves created during the feasibility test in wood for $\gamma$-rays depositing energies $\left(\mathrm{E}_{\gamma}\right)$ between 3 and $4 \mathrm{MeV}$ are shown in Fig. 1. Passive backgrounds have been subtracted, and all curves have been scaled to $25 \mu \mathrm{A}$. Uncertainties shown are twice the Poisson standard deviation. ROC curves for the same data sets integrated from 1 to $31 \mathrm{~s}$, along with similar measurements made in the steel-pipe cargo are shown in Fig. 14. The standard deviation in the active background count rate is taken to be the $\sqrt{N}$ Poisson uncertainty, folded together with a $10 \%$ uncertainty for generator power measurement and scaling as determined by the current experiments. The sensitivity goal of $95 \%$ success rate and $0.1 \%$ false alarm rate is marked by a black box in Fig. 14 .

By experimentally assessing the standard deviation in the $\gamma$-ray background count rate, we have determined that the value used previously $(\sigma=2 \sqrt{N}+15 \%)$ $[1,6]$ is overly conservative by a factor of at least 2 . By using the variation $(\sigma=\sqrt{N}+10 \%)$ determined in the present work, the probability for false alarm decreases, and the success rate increases noticeably over the results of the previous error determination for every data set shown. In particular, the measurement of $\mathrm{HEU}$ with $76 \mathrm{~cm}$ of steel between the floor and the HEU sample is now well inside the detection goals, as shown in Fig. 14.

Integrating the wood data from Fig. 1 out to $31 \mathrm{~s}$ shows that, assuming $\sigma=$ $\sqrt{N}+10 \%$, signals for all 4 cargo thicknesses $\left(R_{f}\right)$ are at least $6 \sigma$ above the 


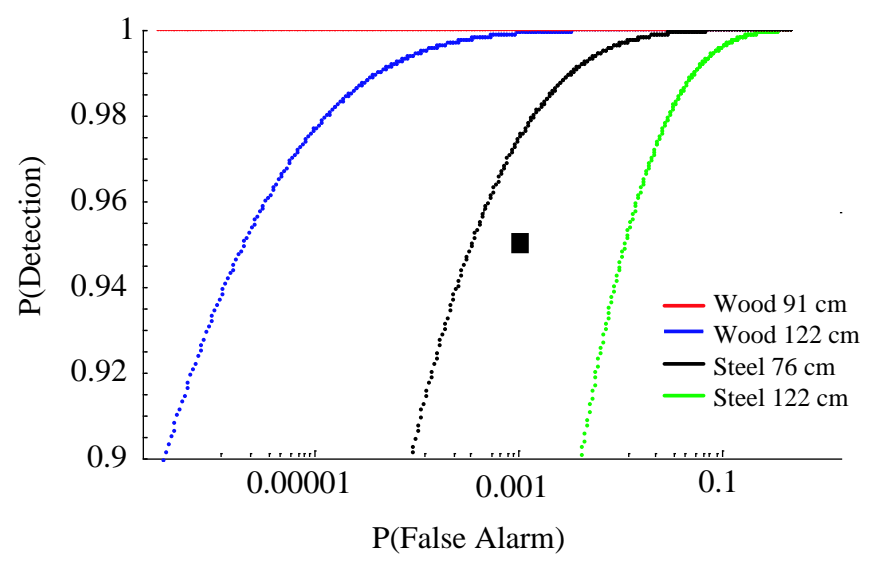

Fig. 14. Receiver Operating Characteristic (ROC) curves for delayed $\gamma$-ray signals in wood and steel cargoes using $\sigma=\sqrt{N}$ folded with $10 \%$ uncertainty in generator power and scaling. The black square indicates 0.95 detection rate and 0.001 false alarm rate. Distances indicated refer to $R_{f}$, and rates have been scaled to a $25 \mu \mathrm{A}$ deuterium current.

active background rate. The goals for $95 \%$ success rate and $0.1 \%$ false alarm rate are therefore met and surpassed at incident neutron attenuation lengths $\left(R_{f}=122 \mathrm{~cm}\right)$ corresponding to the horizontal and vertical center of a standard $610 \times 244 \times 244 \mathrm{~cm}(20 \times 8 \times 8 \mathrm{ft})$ cargo container.

Similar curves have been constructed for the steel mock cargo measurements, also found in Refs. $[1,6]$. Integrating the steel data from 1 to 31 s shows that, again assuming the smaller fluctuation $\sigma=\sqrt{N}+10 \%$, signals for $R_{f}=76 \mathrm{~cm}$ are $5 \sigma$ above the active background count rate, while signals for $R_{f}=122 \mathrm{~cm}$ between the floor and HEU sample are $4 \sigma$ above the active background count rate. The system is sensitive for $0.6 \mathrm{~g} / \mathrm{cm}^{3}$ steel pipe cargo at incident neutron attenuation lengths reaching the horizontal and vertical center of a standard steel cargo container.

\section{Conclusion}

In the Nuclear Car Wash, the radiation signal for fissile material must be detected over both ambient and neutron-activated backgrounds. The background rate has been found to be smaller than assumed in previous reports and projected system performance is thus better than previously estimated. Experiments performed here have shown that the contributions to the background caused by varying generator power, irradiation time and container contents, as well as long term fluctuations in the ambient background do not increase the uncertainty beyond the Poisson statistical standard deviation as 
was previously determined [1,6]. ROC curves constructed with $\sqrt{N}+10 \%$ uncertainty show an increase in the projected success rate and a decrease in the rate of false alarm for every measured cargo configuration. Detection goals of $95 \%$ detection and $0.1 \%$ false alarm are realized for every tested thickness of $0.55 \mathrm{~g} / \mathrm{cm}^{3}$ wood (up to $122 \mathrm{~cm}$ ) and now very clearly for $0.6 \mathrm{~g} / \mathrm{cm}^{3}$ steel pipe cargo up to and including thicknesses of $76 \mathrm{~cm}$. Assuming distributions of the background count rate are Gaussian, it is concluded that the variation due to the influences tested here are within one statistical standard deviation, and that the sensitivity of the Nuclear Car Wash in steel and wood is increased over previous reports $[1,6]$. In terms of areal cargo density, $\rho L$, where $L$ is the incident neutron attenuation length, the system sensitivity extends to objects embedded at $\rho \mathrm{L}=75 \mathrm{~g} / \mathrm{cm}^{2}$. For $98 \%$ of cargoes entering the United States, this corresponds to the horizontal and vertical center of a (610 x $244 \times 244$ $\mathrm{cm})(20 \times 8 \times 8 \mathrm{ft})$ sea-going cargo container.

This work was performed under the auspices of the United States Department of Energy by the University of California, Lawrence Livermore National Laboratory contract number W-7405-Eng-4, UCRL...

\section{References}

[1] J.A. Church et al., Nucl. Instr. and Meth. A, CAARI proceedings (2006).

[2] D.R. Slaughter et al., Nucl. Instr. and Meth. B 241, 777 (2005).

[3] D.R. Slaughter et al., LLNL Technical Report UCRL-ID 155315, (2005).

[4] J.Pruet et al., Journal of Appl. Physics 97, 94908 (2005).

[5] J.Hall et al., Nucl. Instr. and Meth. A, CAARI proceedings (2006).

[6] J.A. Church et al., LLNL Technical Report UCRL-TR-225709, (2006).

[7] E.B. Norman, et al., Nucl. Instr. and Meth. A 521, 608 (2004).

[8] National Nuclear Data Center, www.nndc.bnl.gov.

[9] T.Luu et al., LLNL Technical Report UCRL-TR-227515, (2006).

[10] D. Petersen et al., LLNL Technical Report UCRL-ID pending.

[11] M.-A. Descalle, et al., LLNL Technical Report UCRL-TR-225708, (2006).

[12] M.-A. Descalle, LLNL, private communication, (2006). 